\title{
ON CLOSED IMAGES OF PERFECT PREIMAGES OF ORTHOCOMPACT DEVELOPABLE SPACES
}

\author{
By \\ Takemi MizoKami
}

\section{Introduction.}

We consider the following property of the closed images of topological spaces : For spaces $X, Y$ and a closed mapping $f: X \rightarrow Y$, the following (*) holds:

(*) $Y=Y_{o} \cup \cup\left\{Y_{n}: n \in \omega\right\}$, where $f^{-1}(y)$ is compact for each $y \in Y_{o}$ and $Y_{n}$ is closed and discrete in $Y$ for each $n \in \omega$.

Originally, Lašnev showed in [7] that (*) holds for a metric space $X$, and the other cases are listed in [2, pp. 13 and 14]. A few years ago, Chaber proved that (*) holds for a regular $\sigma$-space $X[3$, Theorem 1.1], and he proposed there the problem whether $\left(^{*}\right)$ holds or not for the cases when $X$ is a perfect preimage of a regular $\sigma$-space or of a Moore space [3, Problems 1.1 and 3.1]. In this paper, we give a characterization of orthocompact developable spaces and give a partial answer to the latter case. We denote the set of all natural numbers by $\omega$. All spaces are assumed to be $T_{1}$. All mappings are assumed to be continuous and onto.

\section{The main results.}

In the sequel, we denote by $[X, Y, Z, f, g]$ the situation that $X, Y, Z$ are spaces, $f: X \rightarrow Y$ is a closed mapping and $g: X \rightarrow Z$ is a perfect mapping. Moreover, we denote by $[X, Y, f]$ the situation that $X, Y$ are spaces and $f: X \rightarrow Y$ is a closed mapping.

Before stating a positive result for some subclass of perfect preimages of Moore spaces, we give the definition of $\mathcal{F}$-preserving families in both sides, which is used to characterize the class of stratifiable $\mu$-spaces by Junnila and the author [6].

DEFINITION 2.1. Let $U, \mathscr{F}$ be families of a space $X$. We call that $u$ is

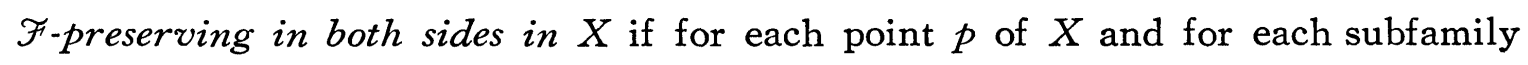
$U_{0}$ of $U$, the following two conditions are satisfied:

(1) If $p \in \cap U_{0}$, then $p \in F \subset \cap U_{0}$ for some $F \in \mathscr{F}$.

(2) If $p \in X-\cup U_{0}$, then $p \in F \subset X-\cup U_{0}$ for some $F \in \mathscr{F}$.

Received July 28, 1986. Revised October 3, 1986. 
$U$ is called $\sigma$-F゙-preserving if $U=U\left\{U_{n}: n \in \omega\right\}$, where each $U_{n}$ is $\mathscr{F}$-preserving in both sides in $X$.

According to Brandenburg [1], a developable space can be characterized as a space which has a $\sigma$-dissectable base, where a family $U=\left\{U_{\alpha}: \alpha \in A\right\}$ of subsets of a space $X$ is called dissectable if for each $\alpha \in A$ there exists a sequence $\left\{D_{\alpha n}: n \in \omega\right\}$ of closed subsets of $X$ satisfying the following:

(1) $U_{\alpha}=\cup\left\{D_{\alpha n}: n \in \omega\right\}$ for each $\alpha \in A$.

(2) For each $n,\left\{D_{\alpha n}: \alpha \in A\right\}$ is closure-preserving in $X$.

(3) For each $n$ and each point $p \in \cup\left\{D_{\alpha n}: \alpha \in A\right\}, \cap\left\{U_{\alpha}: \alpha \in A\right.$ and $\left.p \in D_{\alpha n}\right\}$ is a neighborhood of $p$ in $X$.

We give here a similar characterization of orthocompact developable spaces. To do so, we introduce the notion of $\mathcal{O}$-dissectable families as modified one.

DEFINITION 2.2. Let $X$ be a space and $u$ a family of subsets of $X$. We call $U \mathcal{O}$-dissectable if there exists a $\sigma$-discrete family $\mathscr{F}$ of closed subsets of $X$ satisfying the following:

(1) $U$ is $\mathscr{F}$-preserving in both sides in $X$.

(2) For each $F \in \mathscr{F}, \cap\{U \in U: F \subset U\}$ is a neighborhood of $F$ in $X$, if it is not empty.

LEMMA 2.3. If $U$ is an $\mathcal{O}$-dissectable family of subsets of a space $X$, then $U$ is dissectable.

PROOF. Let $U=\left\{U_{\alpha}: \alpha \in A\right\}$ and $\mathscr{F}=\cup\left\{\mathscr{F}_{n}: n \in \omega\right\}$ with each $\mathscr{F}_{n}$ discrete be the same families of the above definition. For each $\alpha \in A$, set

$$
D_{\alpha n}=\cup\left\{F \in \mathscr{F}_{n}: F \subset U_{\alpha}\right\}, n \in \omega .
$$

Then $\left\{D_{\alpha n}: n \in \omega\right\}, \alpha \in A$, satisfy the required conditions.

LEMMA 2.4. For a family $U$ of subsets of a space $X, U$ is 0 -dissectable if and only if $U$ is interior-preserving and $\mathscr{F}$-preserving in both sides in $X$ for some $\sigma$-discrete family $\mathscr{F}$ of closed subsets of $X$.

ProOF. Only if part: Assume that $U$ and $\mathscr{F}$ satisfy the conditions (1) and (2) of Definition 2.2. To see that $U$ is interior-preserving in $X$, let $p \in \cap U_{0}$ for $u_{o} \subset U$. There exists $F \in \mathscr{F}$ such that $p \in F \subset \cap U_{o}$. By (2), $\cap U_{o}$ is a neighborhood of $p$ in $X$, implying that $\cap U_{o}$ is open in $X$. If part is trivial.

LEMMA 2.5. Let $X$ be an orthocompact developable space. Then each open cover of $X$ has an $\mathcal{O}$-dissectable open refinement.

PROOF. It suffices to show that each interior-preserving open cover of a semi- 
stratifiable space is $\mathscr{F}$-preserving in both sides in $X$ for some $\sigma$-discrete family $\mathscr{F}$ of closed subsets of $X$. Then it is $\mathcal{O}$-dissectable by the above lemma. Let $u=$ $\left\{U_{\alpha}: \alpha \in A\right\}$ be an interior-preserving open cover of $X$. For each point $p \in X$, let $\delta(p)=\left\{\alpha \in A: p \in U_{\alpha}\right\}$ and let $\Delta=\{\delta(p): p \in X\}$. For each $\delta \in \Delta$ and $k \in \omega$, set

$$
F(k, \delta)=\left(\cap\left\{U_{\alpha}: \alpha \in \delta\right\}\right)_{k}-\cup\left\{U_{\alpha}: \alpha \in A-\delta\right\},
$$

where $\left\{\left(\cap\left\{U_{\alpha}: \alpha \in \delta\right\}\right)_{k}: k \in \omega\right\}$ is the semi-stratifiability of an open subset $\cap\left\{U_{\alpha}\right.$ : $\alpha \in \delta\}$. Set

$$
\mathscr{F}(k)=\{F(k, \delta): \delta \in \Delta\}, k \in \omega .
$$

Then $\mathscr{F}=U\{\mathscr{F}(k): k \in \omega\}$ is a $\sigma$-discrete family of closed subsets of $X$ and it is easy to see that $U$ is $\mathscr{F}$-preserving in both sides in $X$. This completes the proof.

THEOREM 2.6. For a space $X$, the following are equivalent:

(1) $X$ is an orthocompact developable space.

(2) $X$ has a $\sigma$-discrete family $\mathscr{F}^{\prime}$ of closed subsets and has a base $\cup\left\{\mathcal{V}_{n}: n \in \omega\right\}$,

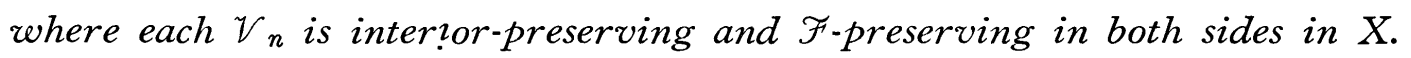

(3) X has a $\sigma-\mathcal{O}$-dissectable base.

ProOF. (1) $\rightarrow(2):$ Let $\left\{U_{n}: n \in \omega\right\}$ be a development for $X$. By the above lemma, for each $n$ there exists a $\sigma$-discrete family $\mathscr{F}_{n}$ of closed subsets of $X$ such that $U_{n}$ has an open refinement $\mathscr{V}_{n}$ such that $\mathcal{V}_{n}$ is $\mathscr{F}_{n}$-preserving in both sides and interior-preserving in $X$. Letting $\mathscr{F}^{\prime}=\cup\left\{\mathscr{F}_{n}: n \in \omega\right\}$ we have the required base $\cup\left\{\mathcal{V}_{n}: n \in \omega\right\}$.

(2) $\rightarrow$ (3) follows directly from Lemma 2.4.

$(3) \rightarrow(1):$ By Lemma 2.3, $X$ has a $\sigma$-dissectable base. Therefore $X$ is developable by [1]. By Lemma 2.4, every open cover of $X$ has a $\sigma$-interior-preserving open refinement. The countable metacompactness of $X$ implies that every open cover of $X$ has an interior-preserving open refinement, i.e., $X$ is orthocompact. This completes the proof.

COROLLARY 2.7. Every orthocompact developable space has a $\sigma$ - F-preserving base for some $\sigma$-discrete family $\mathscr{F}$ of closed subsets of it.

LEMMA 2.8. [11, Lemma 5.4]. Let $\Im$ F be a hereditarily closure-preserving family of closed subsets of a space $Y$. For each $n \in \omega$, let

$Y_{n}=\cup\left\{F_{1} \cap \cdots \cap F_{n}: F_{1}, \cdots, F_{n} \in \mathcal{F}\right.$ and $F_{1} \cap \cdots \cap F_{n}$ is a non-empty finite subset of $Y$ \}.

Then each $Y_{n}$ is closed and discrete in $Y$.

We state the main result. 
THEOREM 2.9. If in $[X, Y, Z, f, g] Z$ is an orthocompact Moore space, then (*) holds.

PROOF. By virtue of Corollary 2.7, it suffices to show that if in $[X, Y, Z, f, g]$ $Z$ is a regular space which has a $\sigma$ - $F$-preserving base for some $\sigma$-discrete (more generally, $\sigma$-locally finite) family $\mathscr{F}$ of closed subsets of $Z$, then $\left(^{*}\right)$ holds.

Let $U=\cup\left\{U_{n}: n \in \omega\right\}$ be a base for $Z$, where each $U_{n}$ is $\mathscr{F}$-preserving in both sides in $Z$. Let $\mathscr{F}=\cup\left\{\mathscr{F}_{n}^{\prime}: n \in \omega\right\}$, where each $\mathscr{F}^{\prime} n^{\prime}$ is a locally finite closed cover of $Z$. For each $n$, let $\mathscr{F}_{n}$ be the totality of finite intersections of members of $\cup\left\{\mathscr{F}_{i} i^{\prime} i \leqq n\right\}$. Then $\left\{\mathscr{F}_{n}: n \in \omega\right\}$ is a sequence of locally finite and finitely multiplicative closed covers of $Z$ such that $\mathscr{F}_{n} \subset \mathscr{F}_{n+1}$ for each $n$. Obviously, each $u_{n}$ is $U_{n} \mathscr{F}_{n}$-preserving in both sides in $Z$ and $U_{n} \mathscr{F}_{n}$ is a network for $Z$. Thus, we can assume $u_{n} \subset U_{n+1}$ for each $n$. For each $n$, write

$$
\mathcal{E}_{n}=g^{-1}\left(\mathscr{F}_{n}\right)=\left\{E_{\lambda}: \lambda \in \Lambda_{n}\right\} \text {. }
$$

For each $n, k \in \omega$, let $\Delta_{n}(k)$ be the totality of subsets $\delta$ of $\Lambda_{n}$ such $|\delta|=k$ and

$$
Y(\delta)=\bigcap\left\{f\left(E_{\lambda}\right): \lambda \in \delta\right\}
$$

is a non-empty finite subset of $Y$. By Lemma 2.8,

$$
Y_{n}(k)=\cup\left\{Y(\delta): \delta \in \Delta_{n}(k)\right\}
$$

is closed and discrete in $Y$. Set

$$
Y_{o}=Y-U\left\{Y_{n}(k): n, k \in \omega\right\} \text {. }
$$

We shall show that for each $y \in Y_{o}, f^{-1}(y)$ is compact in $X$. To do it, we establish the following claims:

Claim 1: For each $n \in \omega$,

$$
\mathcal{E}_{n}(y)=\left\{E \in \mathcal{E}_{n}: E \cap f^{-1}(y) \neq \phi\right\}
$$

is finite.

To see it, assume the contrary, i.e., that for some $m, \mathcal{E}_{m}(y)$ is infinite. Choose an infinite sequence $\left\{E_{m}, E_{m+1}, \cdots\right\} \subset \mathcal{E}_{m}(y)$ and $x_{o} \in f^{-1}(y)$. Observe that for each $k$

$$
E_{k}{ }^{\prime}=\cap\left\{E \in \mathcal{E}_{k}: x_{o} \in E\right\} \in \mathcal{E}_{k} .
$$

Since $y \in Y_{o}, f\left(E_{k}{ }^{\prime}\right) \cap f\left(E_{k}\right)$ is infinite for each $k \geqq m$, we can choose a sequence $\left\{y_{k}: k \geqq m\right\}$ of distinct points of $Y$ such that

$$
y_{k} \in f\left(E_{k^{\prime}}\right) \cap f\left(E_{k}\right), k \geqq m .
$$

Choose two points $p_{k}, p_{k^{\prime}} \in X$ for each $k \geqq m$ such that

$$
p_{k} \in f^{-1}\left(y_{k}\right) \cap E_{k} \text { and } p_{k^{\prime}} \in f^{-1}\left(y_{k}\right) \cap E_{k}{ }^{\prime}
$$

for each $k$. Recall that $\cup\left\{\mathcal{E}_{n}: n \in \omega\right\}$ is a $\Sigma$-network for $Y$ in the sense of Nagami [8]. Therefore, $\left\{p_{k}{ }^{\prime}\right\}$ has a cluster point in $Y$. So, $\left\{y_{k}: k \geqq m\right\}$ consequently has 
a cluster point in $Y$. But this is a contradiction because $\left\{p_{k}: k \geqq m\right\}$ is discrete in $X$ and $f$ is a closed mapping. Hence $\mathcal{E}_{n}(y)$ is finite for each $n$. (The proof of this part have been done referring to [12, Theorem 1.3].)

Claim 2: $g\left(f^{-1}(y)\right)$ is Lindelöf.

In fact, by Claim 1 , for each $n$

$$
\mathscr{F}_{n}(y)=\left\{F \in \mathscr{F}_{n}: g^{-1}(F) \in \mathcal{E}_{n}(y)\right\}
$$

is finite. It is obvious that

$$
\cup\left\{\mathscr{F}_{n}(y): n \in \omega\right\} / g\left(f^{-1}(y)\right)
$$

is a countable network for the subspace $g\left(f^{-1}(y)\right)$. This implies that $g\left(f^{-1}(y)\right)$ is Lindelöf.

Claim 3: There exists a sequence $\left\{y_{n}: n \in \omega\right\}$ of points of $Y$ satisfying the following :

(1) $E \cap f^{-1}\left(y_{n}\right) \neq \phi$ for each $E \in \mathcal{E}_{k}(y)$ and $n \geqq k$.

(2) If $N \subset \omega$ is infinite, then $\left\{y_{n}: n \in N\right\}$ has a cluster point in $Y$.

In fact, by Claim 1 , each $\mathcal{E}_{n}(y)$ is finite. Since $y \in Y_{0}$ and

$$
y \in \cap\left\{f(E): E \in \mathcal{E}_{n}(y)\right\},
$$

$\cap\left\{f(E): E \in \mathcal{E}_{n}(y)\right\}$ is infinite. Thus, we can choose a sequence $\left\{y_{n}: n \in \omega\right\}$ of points of $Y$ such that for each $n$

$$
y_{n+1} \in \cap\left\{f(E): E \in \mathcal{E}_{n+1}(y)\right\}-\left\{y_{1}, \cdots, y_{n}\right\} .
$$

It is obvious to see that $\left\{y_{n}: n \in \omega\right\}$ satisfies (1). Let $N$ be an infinite subset of $\omega$. Since for a point $x_{0} \in f^{-1}(y)$,

$$
E_{n}{ }^{\prime}=\cap\left\{E \in \mathcal{E}_{n}: x_{o} \in E\right\} \in \mathcal{E}_{n}(y), n \in N,
$$

there exists by Claim 3(1),

$$
p_{n} \in f^{-1}\left(y_{n}\right) \cap E_{n}^{\prime}, n \in N .
$$

By the same reason as in the proof of Claim 1, $\left\{y_{n}: n \in N\right\}$ has a cluster point in $Y$.

Finally we show that $f^{-1}(y)$ is compact in $X$. Assume that $f^{-1}(y)$ is not compact in $X$. Then $g\left(f^{-1}(y)\right)$ is not so in $Z$ because $g$ is a perfect mapping. Recall that by Claim $2 g\left(f^{-1}(y)\right)$ is Lindelö. By the argument of [3, Theorem 1] there exists an increasing open cover $\left\{U_{i}: i \in \omega\right\}$ of $g\left(f^{-1}(y)\right)$ such that for each $i$

$$
g\left(f^{-1}(y)\right) \cap\left(U_{i+1}-\bar{U}_{i}\right) \neq \phi .
$$

Take points $p_{1} \in U_{1}$ and

$$
p_{i+1} \in g\left(f^{-1}(y)\right) \cap\left(U_{i+1}-\bar{U}_{i}\right)
$$


for each $i$. Set

$$
A_{i}=Z-\cup\left\{U \in U_{i}: U \cap g\left(f^{-1}(y)\right)=\phi\right\}
$$

for each $i$. Then $\left\{A_{i}: i \in \omega\right\}$ is a decreasing sequence of closed subsets of $Z$ such that

$$
g\left(f^{-1}(y)\right)=\cap\left\{A_{i}: i \in \omega\right\} .
$$

Since $U_{i}$ is $\cup_{k} \mathscr{F}_{k}$-preserving in both sides in $Z$, there exists $F_{i} \in \cup_{k} \mathscr{F}_{k}$ such that $p_{1} \in F_{1} \subset U_{1}$ and

$$
p_{i_{+1}} \in F_{i_{+1}} \subset\left(U_{i_{+1}}-\bar{U}_{i}\right) \cap A_{i} .
$$

By Claim 3 (1), we can choose $\left\{y_{n(i)}: i \in \omega\right\}$ such that

$$
F_{i} \cap g\left(f^{-1}\left(y_{n(i)}\right)\right) \neq \phi \text { and } n(i)<n(i+1)
$$

for each $i$. If we take for each $i$

$$
x_{i} \in g^{-1}\left(F_{i}\right) \cap f^{-1}\left(y_{n(i)}\right),
$$

then by Claim $3(2),\left\{g\left(x_{i}\right): i \in \omega\right\}$ has a cluster point $z$ in $Z$. Since $g\left(x_{i}\right) \in F_{i}$, $i \in \omega$, and $\left\{F_{i}: i \in \omega\right\}$ is discrete in the subspace $g\left(f^{-1}(y)\right), z$ must belong to $Z-$ $g\left(f^{-1}(y)\right)$. Since $g\left(f^{-1}(y)\right)=\cap\left\{A_{i}: i \in \omega\right\}$, there exists $m \in \omega$ such that $z \notin A_{n}$ for every $n \geqq m$. But this is a contradiction because $g\left(x_{n}\right) \in A_{m}$ for every $n \geqq m$ and $A_{m}$ is closed in $Z$. Hence we have shown that $f^{-1}(y)$ is compact in $X$. This completes the proof.

From here, we assume that all $p$-spaces are regular. In [4], Filippov showed that $\left(^{*}\right)$ holds if $X$ is a paracompact $p$-space in $[X, Y, f]$. We generalize it as follows :

COROllaRY 2.10. If in $[X, Y, f] X$ is an orthocompoct, d-paracompact p-space, then (*) holds.

Proof. By [9, Theorem 4.4] there exists a perfect mapping of $X$ onto a Moore space $Z$. By [5, Theorems 3.2 and 3.3] $Z$ is orthocompact. Thus, by the theorem $\left(^{*}\right)$ holds.

REMARK. We know that Veličko showed that $\left(^{*}\right)$ holds if $X$ is a metacompact, completely regular $p$-space [13], as a generalization of Filippov's result. But, Corollary 2.10 is not the corollary of Veličko's, because there exists an orthocompact Moore space $X$ which is not metacompact [13, Theorem 2]. 


\section{References}

[1] Brandenburg, H., Some characterizations of developable spaces. Proc. Amer. Math. Soc. 80 (1980), 157-161.

[2] Burke, D. K., Closed mappings. Surveys in general topology, Academic press, 1980.

[ 3 ] Chaber, J., Generalizations of Lašnev theorems. Fund. Math. 119 (1983), 85-91.

[4] Filippov, V. V., On feathered paracompacta. Soviet Math. Dokl. 9 (1968), 161-164.

[5] Gruenhage, G., On closed images of orthocompact spaces. Proc. Amer. Math. Soc. 77 (1979), 389-394.

[6] Junnila, H. J. K. and Mizokami, T., Characterizations of stratifiable $\mu$-spaces. Topology Appl. 21 (1985), 51-58.

[7] Lašnev, N., Continuous decompositions and closed mappings of metric spaces. Soviet Math. Dokl. 6 (1965), 1504-1506.

[8] Nagami, K., $\Sigma$-spaces. Fund. Math. 55 (1969), 169-192.

[9] Pareek, C. M., Moore spaces, semi-metric spaces and continuous mappings connected with them. Canad. J. Math. 24 (1972), 1033-1042.

[10] Reed, G. M., On the existence of point countable bases in Moore spaces. Proc. Amer. Math. Soc. 45 (1974), 432-440.

[11] Suzuki, J., On pre- $\sigma$-spaces. Bull. Tokyo Gakugei University Ser. 28 (1976), 22-23.

[12] Tanaka, Y. and Yajima, Y., Decompositions for closed maps, preprint.

[13] Veličko, N. V., On $p$-spaces and their continuous maps. Math. USSR Sbornik 19 (1973), $35-46$.

Joetsu University of Education

Joetsu, Niigata 943

Japan 